\title{
Ising-like model for the two step spin-crossover of binuclear molecules
}

\author{
A. Bousseksou $\left({ }^{1,2}\right)$, F. Varret $\left({ }^{1}\right)$ and J. Nasser $\left(^{1}\right)$ \\ (') Département de Recherches Physiques (*), Université Pierre et Marie Curie, 75252 Paris \\ Cedex 05, France \\ (2) Laboratoire de Chimie de Coordination du CNRS, 205 route de Narbonne, 31077 Toulouse \\ Cedex, France
}

(Received 29 July 1992, revised 3 December 1993, accepted 18 February 1993)

\begin{abstract}
We present a two-sublattice Ising-type model, involving one intramolecular interaction treated rigorously and intermolecular interactions treated in the mean-field approach, in order to describe the two-step transition exhibited by a bi-nuclear spin-crossover complex. Spontaneous symmetry breakings can be obtained according to the relative values of the interactions parameters. The phase diagram is given and the experimental data of $[\mathrm{Fe}(\mathrm{bt})$ $\left.(\mathrm{NCS})_{2}\right]_{2}$ bpym are treated in the present model.
\end{abstract}

\section{Introduction.}

At variance from the usual « one-step» spin-crossover transition, a few mononuclear $\mathrm{Fe}^{\mathrm{Il}}$ complexes exhibit a two-step conversion curve $n_{\mathrm{HS}}(T)$ (where $n_{\mathrm{HS}}$ is the high spin proportion), with a plateau of some kelvin [1-4]. Such one- and two-step transitions have been modelized in the discrete level formalism, as $N$ interacting two-level systems [5-8] : using an "Ising-like " model, treated in the mean-field approximation with two « ferromagnetically coupled » sublattices, " antiferromagnetically » coupled to each other, we could adequately reproduce the typical shapes of two-step conversions [8].

We recently observed a two-step conversion in a $\mathrm{Fe}^{\mathrm{II}}$ binuclear complex [9] and we report here an extension of the previous Ising-like treatment, where the intramolecular (bimetallic) Hamiltonian is rigorously treated; this follows the conclusions of the Mössbauer study [9] showing that the macroscopic two-step transition closely reflects the following intramolecular steps :

$$
\text { LS-LS } \rightleftharpoons(\text { LS-HS or HS-LS }) \rightleftharpoons \text { HS-HS . }
$$

(*) UA CNRS n 71 . 
We briefly discuss other previous two-step models :

(i) In [9] some of us proposed an extension of the thermodynamical approach of non-regular solutions (following Drickamer's model [10] for one-step transitions). In such an approach the molecular states HS-LS and LS-HS are indistinguishable ; this assumption is inherent to the description of the system in terms of a fluid, and is certainly questionable in a crystal : the interaction energy of a given pair of molecules depends on their orientations in the crystal, and for instance the energies of the sequences (HS-LS)-(HS-LS) and (HS-LS)-(LS-HS) a priori are not equal. In other words, the " fluid " description discards the eventuality of an ordered ferroelectric phase.

(ii) On the contrary, the two-sublattice approach followed in references [7, 8] (Ising-like) and [11] (cooperative distortions with an explicit description of the interactions) can account for the anisotropic effects in the crystalline state. Both kinds of models result in similar sets of conversion curves with similar symmetry breakings : figures 2,3 of reference [11] compare very closely to figure 11 of reference [8]. We did not try to adapt the already complex model of reference [11], since the resolution of a similar problem of magnetism was given a long time ago by Oguchi [12] in the suited Ising formalism.

\section{Formalism.}

The Hamiltonian of an isolated metallic center is :

$$
\hat{\mathcal{X}}=\frac{1}{2} \delta \hat{\sigma},
$$

where $\hat{\sigma}$ is a fictitious spin operator with eigenvalues \pm 1 associated with high spin (HS) and low-spin (LS) states of the metal. The degeneracies of the eigenvalues are such that $g_{\mathrm{LS}} \neq g_{\mathrm{HS}}$, because of the different electronic and vibrational properties of the spin states [11]. The ratio of high-spin state $n_{\mathrm{HS}}(T)$ and the thermal average of the fictitious spin are related by:

$$
n_{\mathrm{HS}}=(1+(\hat{\sigma})) / 2 \text {. }
$$

At the solid state, electronic and steric effects result in interactions between the metallic centers. The pair interaction is developed as :

$$
W_{1 j}=A_{i j}\left[\dot{\sigma}_{i}+\dot{\sigma}_{j}\right]+J_{1 j} \hat{\sigma}_{1} . \hat{\sigma}_{j},
$$

so that the Hamiltonian is :

$$
\hat{\mathfrak{H}}=\sum_{1} \frac{1}{2} \Delta_{1} \hat{\sigma}_{1}+\sum_{\langle i, j\rangle} J_{1 j} \hat{\sigma}_{1} . \hat{\sigma}_{j} .
$$

Both $\Delta_{1}$ and $J_{1 j}$ are phenomenological parameters : $\Delta_{i}$ is the energy splitting of the uncoupled two-level system $i$, and $J_{1}$ are interaction parameters.

As in [8], for simplicity we consider $\Delta_{i}=\Delta$ at all sites, and here we specifically consider a crystal containing one symmetric bimetallic molecule in each elementary cell, in such a position that the metal positions form two equivalent sublattices $A$ and $B$.

For a better understanding of the present results, we briefly recall some results of the previous mean-field model $[8,13]$ in the case of equivalent sublattices. 


\section{Basic results of the previous mean-field treatment.}

Two one-site Hamiltonians are defined :

$$
\begin{aligned}
& \hat{\mathcal{H}}_{\mathrm{A}}=\frac{\Delta}{2} \cdot \hat{\sigma}_{\mathrm{A}}+\hat{\sigma}_{\mathrm{A}}\left[J\left[\hat{\sigma}_{\mathrm{A}}\right]+J_{\mathrm{AB}}\left\langle\hat{\sigma}_{\mathrm{B}}\right\rangle\right] \\
& \hat{\mathcal{C}}_{\mathrm{B}}=\frac{\Delta}{2} \cdot \hat{\sigma}_{\mathrm{B}}+\hat{\sigma}_{\mathrm{B}}\left[J\left[\hat{\sigma}_{\mathrm{B}}\right]+J_{\mathrm{AB}}\left\langle\hat{\sigma}_{\mathrm{A}}\right\rangle\right]
\end{aligned}
$$

where the interaction parameters, $J<0 \ll$ ferromagnetic » and $J_{\mathrm{AB}}>0$ 《antiferromagnetic », account for the number of neighbours.

The mean-field equations are easily derived :

$$
\begin{aligned}
& \left\langle\dot{\sigma}_{\mathrm{A}}\right\rangle=f_{\mathrm{A}}\left(\left\langle\hat{\sigma}_{\mathrm{A}}\right\rangle,\left\langle\hat{\sigma}_{\mathrm{B}}\right\rangle, J_{\mathrm{A}}, J_{\mathrm{AB}}\right) \\
& \left\langle\hat{\sigma}_{\mathrm{B}}\right\rangle=f_{\mathrm{B}}\left(\left\langle\dot{\sigma}_{\mathrm{A}}\right\rangle,\left\langle\hat{\sigma}_{\mathrm{B}}\right\rangle, J_{\mathrm{A}}, J_{\mathrm{AB}}\right) .
\end{aligned}
$$

A fully analytical treatment of equations (3) yields :

i) as in [6], the «trivial " solution $\left\langle\hat{\sigma}_{\mathrm{A}}\right\rangle=\left\langle\hat{\sigma}_{\mathrm{B}}\right\rangle=0$ is obtained at $T=T_{\mathrm{c}}$ defined by $k T_{\mathrm{c}}=\Delta \operatorname{Ln}(r)$ (where $\left.r=g_{\mathrm{HS}} / g_{\mathrm{LS}}\right)$;

ii) for $J_{\mathrm{AB}}$ larger than a threshold value, a spontaneous symmetry breaking occurs within a temperature range limited by two Néel temperatures $\left(\left\langle\hat{\sigma}_{\mathrm{A}}(T)\right\rangle \neq\left\langle\hat{\sigma}_{\mathrm{B}}(T)\right\rangle\right.$ for $T_{\mathrm{N}}^{-}<$ $\left.T<T_{\mathrm{N}}^{*}\right)$

iii) a symmetry breaking is also associated with a continuous or discontinuous variation of the high-spin proportion $n_{\mathrm{HS}}(T)$; in the former case, it is accompanied by a discontinuity of the specific heat and henceforth characterizes a second-order phase transition; in the latter case the transition is of first order;

iv) the two-step evolution of the conversion curve $n_{\mathrm{HS}}(T)=\frac{1}{2}+\frac{1}{4}\left(\left\langle\sigma_{\mathrm{A}}(T)\right\rangle+\left\langle\sigma_{\mathrm{B}}(T)\right\rangle\right)$ is tightly related to the onset of symmetry breakings.

It is noteworthy that the above conclusions are in close agreement with those of the cooperative distortion model of reference [11]. A detailed analysis of the Ising-like model and the derived phase diagrams are given in [8].

\section{The present treatment.}

The Hamiltonian of a molecule (symmetric pair of metallic centers) is written :

$$
\begin{aligned}
\hat{\mathscr{H}}_{\mathrm{P}}= & \frac{\Delta}{2}\left(\hat{\sigma}_{\mathrm{A}}+\hat{\sigma}_{\mathrm{B}}\right)+J\left(\hat{\sigma}_{\mathrm{A}}\left\langle\hat{\sigma}_{\mathrm{A}}\right\rangle+\hat{\sigma}_{\mathrm{B}}\left\langle\hat{\sigma}_{\mathrm{B}}\right\rangle\right)+ \\
& +J_{\mathrm{AB}} \hat{\sigma}_{\mathrm{A}} \cdot \hat{\sigma}_{\mathrm{B}}+J^{\prime}\left(\hat{\sigma}_{\mathrm{A}}\left\langle\hat{\sigma}_{\mathrm{B}}\right\rangle+\hat{\sigma}_{\mathrm{B}}\left\langle\hat{\sigma}_{\mathrm{A}}\right\rangle\right)
\end{aligned}
$$

where

$$
\begin{aligned}
J= & J_{\mathrm{AA}}=J_{\mathrm{BB}}<0: \text { intrasublattice interaction }, \\
J^{\prime}= & J_{\mathrm{AB}}^{\mathrm{Intermol}}<0: \text { intersublattice interaction for } \\
& \text { metallic centers belonging to different molecules },
\end{aligned}
$$

$$
J_{\mathrm{AB}}>0 \text { : intramolecular interaction. }
$$

(In the mean-field treatment, the above interactions can be rewritten in terms of only two interaction parameters, intra- and inter-sublattice). 
As in [8] we define two symmetry-adapted parameters :

$$
\begin{gathered}
m=\left\langle\hat{\sigma}_{\mathrm{A}}\right\rangle+\left\langle\hat{\sigma}_{\mathrm{B}}\right\rangle \\
n=\left\langle\hat{\sigma}_{\mathrm{A}}\right\rangle-\left\langle\hat{\sigma}_{\mathrm{B}}\right\rangle .
\end{gathered}
$$

$m$ is directly related to the high-spin fraction :

$$
n_{\mathrm{HS}}=\frac{1}{2}+\frac{m}{4}
$$

$n$ is an order parameter in Landau description : for $n=0$ the sublattices are equivalent ; for $n \neq 0$ the sublattices are no longer equivalent. The onset of $n \neq 0$ at a given temperature describes a spontaneous symmetry breaking of the system, associated with the loss of inversion symmetry for the molecule.

The Hamiltonian of equation (4) is solved in the $\sigma_{\mathrm{A}} \otimes \sigma_{\mathrm{B}}$ space, and the mean-field equations are derived :

$$
\begin{aligned}
& m=\frac{2 r^{2} \exp -\beta\left(\Delta+\left(J+J^{\prime}\right) m\right)-2 \exp \beta\left(\Delta+\left(J+J^{\prime}\right) m\right)}{D(T)} \\
& n=\frac{-4 r \operatorname{sh}\left(\beta\left(J-J^{\prime}\right) n\right) \exp \left(2 \beta J_{\mathrm{AB}}\right)}{D(T)}
\end{aligned}
$$

where :

$$
\begin{aligned}
D(T)=r^{2} \exp -\beta\left(\Delta+\left(J+J^{\prime}\right) m\right)+\exp \beta\left(\Delta+\left(J+J^{\prime}\right) m\right)+ \\
\quad+2 r \exp \left(2 \beta J_{\mathrm{AB}}\right) \operatorname{ch}\left(\beta\left(J-J^{\prime}\right) n\right) .
\end{aligned}
$$

It is worth noting that equations $\left(5,5^{\prime}\right)$ can be rewritten in terms of $J^{\prime}+J=2 J_{\mathrm{av}}$ and $J^{\prime}-J=\Delta J$. These new parameters are better suited, together with $J_{\mathrm{AB}}$, to describe the properties of the system.

Before reporting a detailed investigation of the problem, we show typical results in figure 1 , which allow for deriving the following observations :

(i) the second step of the conversion (on increasing temperatures) is in any case smoother than the first one ; this is similar to the previous mean-field treatment;

(ii) however, the two-step character of the transition is no longer monitored by the symmetry breakings. This is at variance from the previous model. No doubt this difference originates from the indistinguishability of the two moieties of the molecule in the quantum mechanical treatment of the intramolecular interaction. Then the two-step character can only be analyzed through the derivative $\frac{\mathrm{d}}{\mathrm{d} T} n_{\mathrm{HS}}(T)$; there is no simple analytical expression of this derivative, so that the detailed study of the two-step character has been performed numerically ;

(iii) equation (5) clearly shows that the thermal variation $m(T)$ associated with the symmetrical solution ( $n=0$, which is the thermodynamically stable solution in the absence of symmetry breaking) does not depend on $\Delta J$; it of course depends on $J_{\mathrm{av} .}, J_{\mathrm{AB}}$.

\section{Spontaneous symmetry breakings.}

Of major interest is the spontaneous symmetry breaking which can occur between two limiting temperatures $T_{\mathrm{N}}^{-}, T_{\mathrm{N}}^{+}$, denoted « Néel temperatures » because of the similarity with the paraantiferromagnetic transition. It is clear, by reference to the previous mean-field model, that the symmetry breakings are related to the coupling term $J_{\mathrm{AB}}(>0)$. The relationship with 

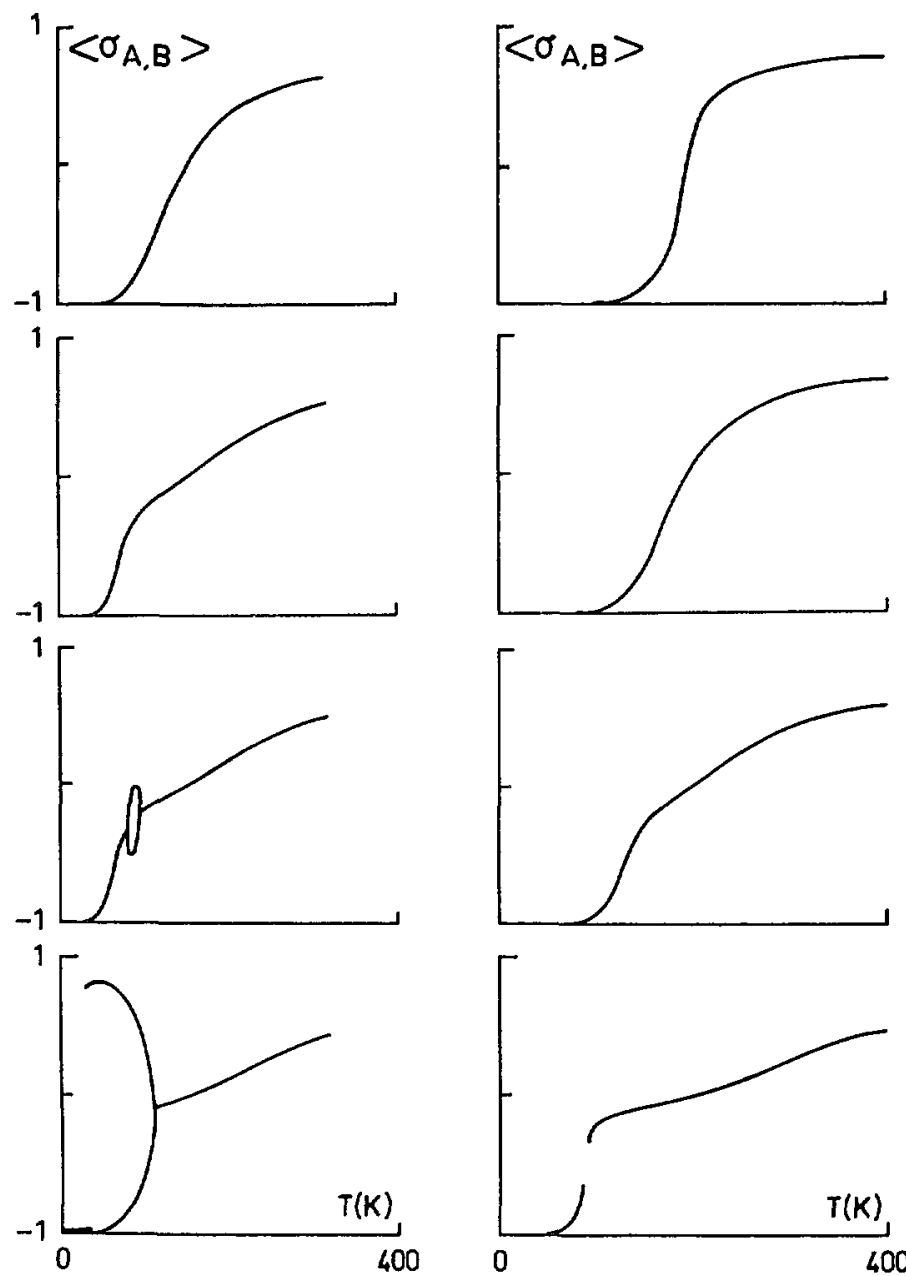

(a)

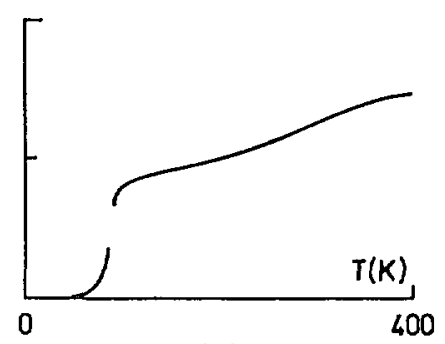

(b)

Fig. 1. - Conversion curves of the two sublattices, $\left\langle\hat{\sigma}_{\mathrm{A}}(T)\right\rangle,\left\langle\hat{\sigma}_{\mathrm{B}}(T)\right\rangle$, computed with $J=$ $-0.4 k T_{\mathrm{c}}$, (a) $J^{\prime}=0, J_{\mathrm{AB}}=0.2,0.7,0.77,1.0 k T_{\mathrm{c}}$; (b) $J^{\prime}=J, J_{\mathrm{AB}}=0.0,0.3,0.6,1.0 k T_{\mathrm{c}}$ (from top to bottom).

$\Delta V$ is examined first :

(i) there is no symmetry breaking for $\Delta J=0$; this is obvious from equation $\left(5^{\prime}\right)$, and can be explained by considering that then the system is no more than a single lattice with «ferromagnetic" interactions plus an intramolecular interaction; this exactly matches the description in terms of thermodynamics of solutions [9]. On the other hand, one can wonder whether the cooperative distortion model [11] might also yield a two-step conversion curve with no symmetry breaking, thanks to an adequate set of values of the interaction parameters ; it seems that a systematical search of the phase diagram has not been performed, so that the question remains open;

(ii) equation $\left(5^{\prime}\right)$ also shows that solutions with $n \neq 0$ require $\Delta J>0$, i.e. $|J|>$ $\left|J^{\prime}\right|$ : otherwise, the two members of the equation have opposite signs; 
(iii) the onset of symmetry breakings requires $\Delta J$ larger than a threshold value (depending on $J_{\mathrm{AB}}, J_{\mathrm{av}}$.). This is demonstrated in the following.

We have developed an original method for calculating such « Néel » temperatures when the variation of the order parameter $n$ is continuous ; then the variation of $m$ is also continuous, and it can be reasonably supposed, in analogy with the previous mean-field model, that this gives rise to a second-order phase transition (the calculation of the specific heat is more complex and has not been undertaken here).

$T_{\mathrm{N}}$ is such that :

$\begin{array}{cc}n\left(T_{\mathrm{N}}\right)=0, & n\left(T_{\mathrm{N}}+\mathrm{d} T\right)=\mathrm{d} n \neq 0 \\ \text { with : } \quad & m\left(T_{\mathrm{N}}\right)=m_{\mathrm{s}}, \quad m\left(T_{\mathrm{N}}+\mathrm{d} T\right)=m_{\mathrm{s}}+\mathrm{d} m,\end{array}$

where $m(T), n(T)$ are solutions of equations $\left(5,5^{\prime}\right)$. The subscript $\mathrm{s}$ denotes that $m_{\mathrm{s}}$ is a symmetrical solution of the system (Eqs. $\left(5,5^{\prime}\right)$ always have a symmetrical solution). Equation (5') is rewritten :

$$
n(T) D(T)=4 r \operatorname{sh}(\beta \Delta J) n(T) \exp \left(2 \beta J_{\mathrm{AB}}\right) .
$$

Then, differentiating equation (6) at $T=T_{\mathrm{N}}$, it comes :

$$
\mathrm{d} n\left(D\left(T_{\mathrm{N}}\right)-4 r \beta_{\mathrm{N}} \Delta J \exp \left(2 \beta_{\mathrm{N}} J_{\mathrm{AB}}\right)\right)=0 .
$$

This equation has a solution $\mathrm{d} n \neq 0$ if :

$$
k T_{\mathrm{N}} D\left(T_{\mathrm{N}}\right) \exp \left(-2 \beta_{\mathrm{N}} J_{\mathrm{AB}}\right)=f\left(T_{\mathrm{N}}\right)=4 r \Delta J
$$

with

$f(T)=k T\left(r^{2} \exp \left(-\beta\left(\Delta+2 J_{\mathrm{av}} m_{\mathrm{s}}+2 J_{\mathrm{AB}}\right)\right)+\exp \left(-\beta\left(-\Delta-2 J_{\mathrm{av},} m_{\mathrm{s}}+2 J_{\mathrm{AB}}\right)\right)+2 r\right)$.

The values of $T_{\mathrm{N}}$ are easily determined by the intersect of the function $f(T)$ and the straight line $g(T)=4 r \Delta J$. The calculation of $f(T)$ implies the resolution of equation (5) for $n=0$, which provides the values of $m_{\mathrm{s}}(T)$. It appears from calculations that $f(T)$ has a grossly parabolic shape, of positive curvature, with a single minimum value positive. Typical plots of $f(T)$ are reported in figure 2 . They lead to a positive threshold value for $\Delta J$, above which two Néel temperatures appear. Of course, if $\Delta J=0$, the threshold value cannot be reached and the system remains symmetrical at all temperatures. This does not prevent the two-step spin conversion, and in this particular case the present model is equivalent to the thermodynamical model of reference [9].

When the symmetry breaking is associated with a discontinuous variation of the order parameter $n$ (and consequently to a discontinuous variation of $m$ ), the first-order Néel temperatures exhibited in figure 1 can be determined only numerically. There are no analytic arguments similar to those developed in the previous mean-field case [8].

\section{Phase diagram.}

Phase diagrams of the 1-2 step and continuous-discontinuous (C-D) characters of the conversion curve, and also of the symmetry breakings have been investigated numerically as a function of $J, J^{\prime}, J_{\mathrm{AB}}$.

Figure 3 illustrates the general case $\Delta J \neq 0$ where symmetry breakings can occur (for simplicity we took $J^{\prime}=0$ ). 


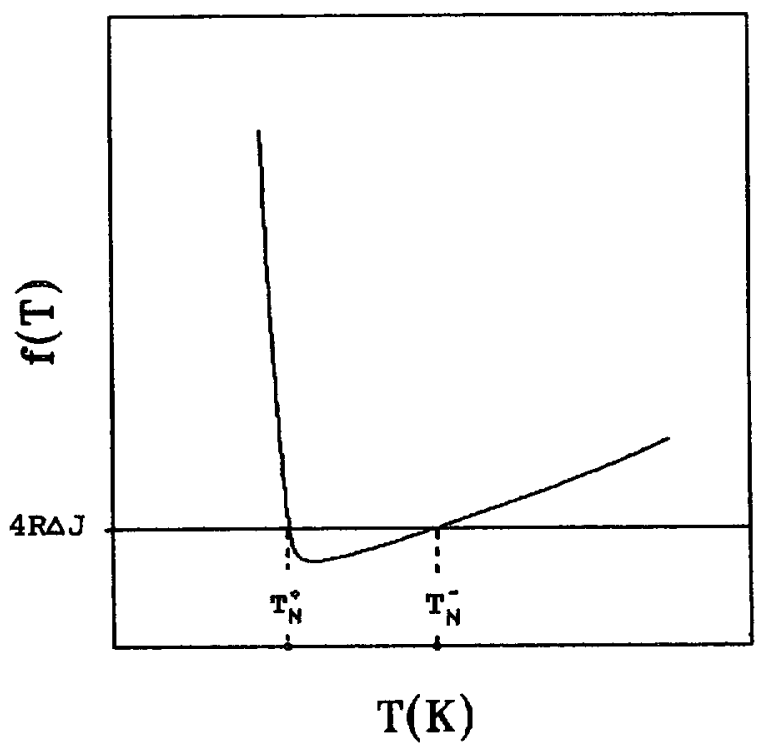

Fig. 2. - A typical $f(T)$ curve for the graphical determination of $T_{\mathrm{N}}^{+}: J_{\mathrm{av}}=-0.25 k T_{\mathrm{c}} ; J_{\mathrm{AB}}=$ $1.1 k T_{\mathrm{c}}$.

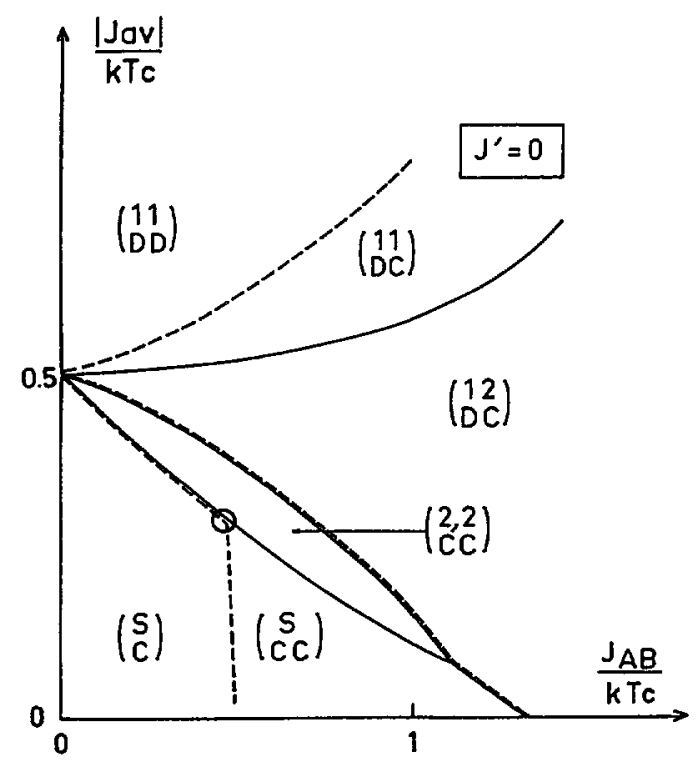

Fig. 3. - Phase diagram of the present model, using $r=\frac{g_{\mathrm{HS}}}{g_{\mathrm{LS}}}=15$, for $J^{\prime}=0$, predicting the characters of the spin conversion: (S): symmetrical at all temperatures: $\left\langle\hat{\sigma}_{\mathrm{A}}(T)\right\rangle=\left\langle\hat{\sigma}_{\mathrm{B}}(T)\right\rangle ;(2,2),(1,2)$, $(1,1)$ order of the Néel transitions which limit the asymmetric phase. (C), (D), (CC), (DC), (DD) continuous or discontinuous character of the one or two steps of the transition. Full lines show symmetry breakings, dashed lines, the characters of the conversion curve. 
Figure 4 illustrates the particular $\Delta J=0$ where symmetry breakings cannot occur; it also holds for any value of $\Delta J$, in $J_{\mathrm{av}}, J_{\mathrm{AB}}$ axes, when the symmetrical solution is considered alone (see Sect. 3 remark (iii)) and therefore can be denoted as the "symmetrical " diagram. The (dashed) borderline which separates $(S, C)$ from $(S, C C)$ in figure 3 is a part of this «symmetrical » diagram.

Starting from the "asymmetric » diagram of figure 3 , by increasing the value of $J^{\prime} / J$ (i.e. decreasing $\Delta J$, the borderlines of the symmetry breakings in figure 3 (full lines) move upwards, so that the area (S) where the phase diagram of figure 4 is relevant, increases progressively so as to extend over the whole plane for $\left|J^{\prime}\right| \geqslant|J|(\Delta J \leqslant 0)$. An intermediate phase diagram is shown in figure 5.

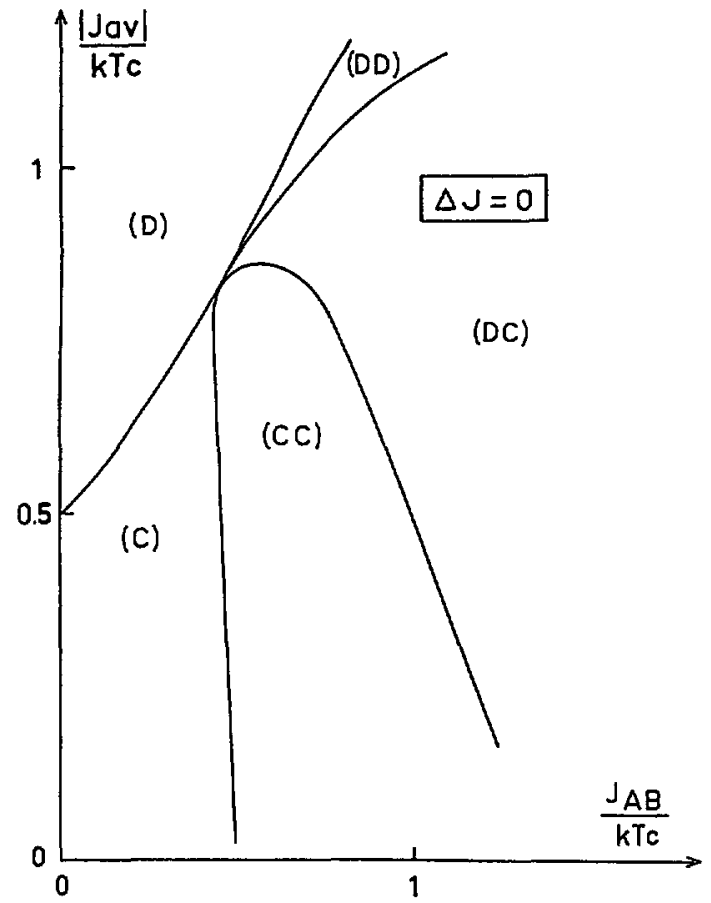

Fig. 4.

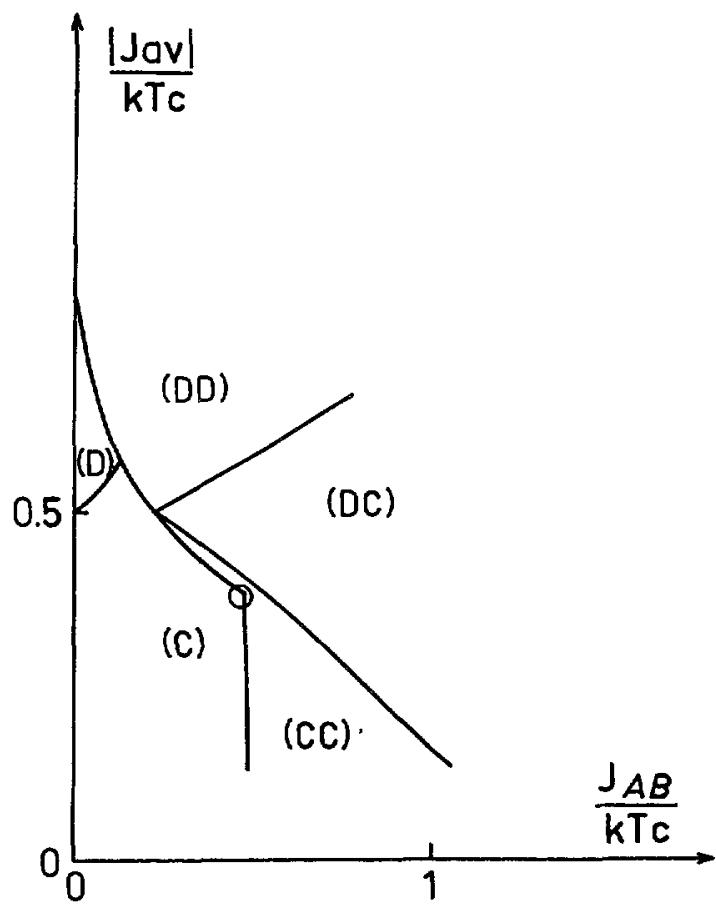

Fig. 5.

Fig. 4. - Phase diagram for the particular case $\Delta J=0$, which also holds for the symmetrical solution in the general case (see text).

Fig. 5. - Phase diagram for $\frac{J^{\prime}}{J}=0.1$.

We have not systematically investigated the threshold value $\Delta J_{\text {thres }}$ as a function of $J_{\mathrm{av}}, J_{\mathrm{AB}}$. However, numerical data obtained so far indicate that an increased $J_{\mathrm{AB}}$ tends to decrease $\Delta J_{\text {thres. }}$; this corresponds to the simple idea that $J_{\mathrm{AB}}$ and $\Delta J$ both favour the occurrence of the symmetry breaking (simple schemes show that $\frac{\Delta J}{2}$ acts as an «antiferromagnetic " intermolecular interaction between $\mathrm{A}, \mathrm{B}$ sublattices). We also found that an increased $\left|J_{\text {av. }}\right|$ led to a decreased $\Delta J_{\text {thres., }}$, indicating that the average «ferromagnetic » interaction also favours the symmetry breakings ; this is the so-called " synergical " effect of the "ferro-" and « antiferro-magnetic » interactions reported in reference [9]. 
It can also be mentioned that $\Delta J_{\text {thres. }}$ is tightly related to the phase diagram : indeed $\Delta J=\Delta J_{\text {thres }}$ corresponds to a situation where the «symmetrical » and «non-symmetrical » phase diagrams intersect each other; the corresponding crossing points are represented by circles in figures 3,5 .

\section{Analysis of the experimental data.}

In figure 6 we show the experimental data $n_{\mathrm{HS}}(T)$ of the bi-nuclear complex $\left(\mathrm{Fe}(\mathrm{bt})(\mathrm{NCS})_{2}\right)_{2}$ bpym (from [9]). In a first fit, the ratio $r$ of the degeneracies was fixed to the « electronic » value $15\left(g_{\mathrm{HS}}=15=(2 L+1)(2 S+1) ; g_{\mathrm{LS}}=1\right) ; \Delta J=0$ was fixed; all other parameters : $J_{\mathrm{av} .}, J_{\mathrm{AB}}, \Delta$ were free. Then, the high-temperature computed $n_{\mathrm{HS}}$ values were far too small; in addition, the computed value of the entropy change per metallic ion $\left(R T \operatorname{Ln}(15)=13.4 \mathrm{~J} . \mathrm{K}^{-1} \mathrm{~mole}^{-1}\right)$ was far below the measured value (41 \pm $3 \mathrm{~J} . \mathrm{K}^{-1}$ mole $^{-1}$ ) [9]).

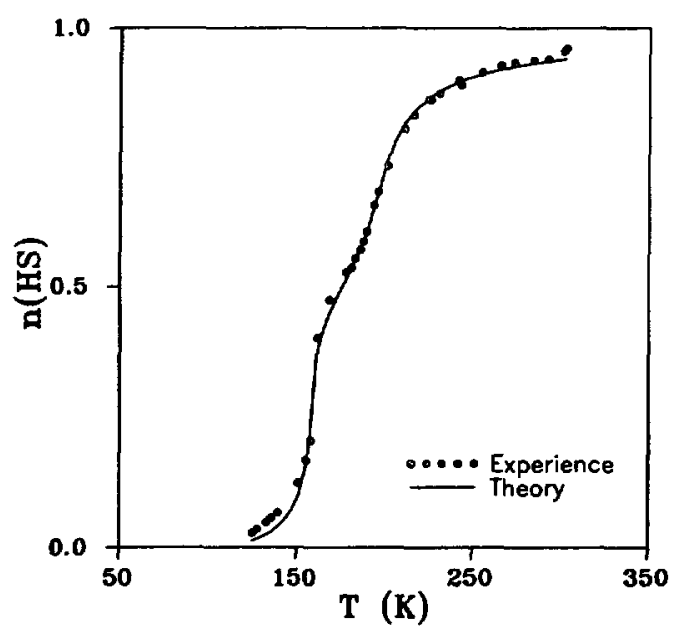

Fig. 6. - Best fit of the conversion curve of $\left[\mathrm{Fe}(\mathrm{bt})(\mathrm{NCS})_{2}\right]_{2}$ bpym, using the present model (data in Tab. I).

To explain this discrepancy, we recently showed [13], that the account of intramolecular vibrations in the model allowed this ratio to be modified at the high-temperature limit: it becomes $\left(g_{\mathrm{HS}} / g_{\mathrm{LS}}\right) \times\left(\omega_{\mathrm{LS}} / \omega_{\mathrm{HS}}\right)$, where $\omega_{\mathrm{LS}}, \omega_{\mathrm{HS}}$ are the intramolecular vibration frequencies and sizably differ in the low and high spin states (by a factor of two at least, according to IR data [14]).

So, we have fitted again the conversion curve by considering $r$ as a free parameter. The hightemperature values are now well reproduced, and the calculated value of the molar entropy change upon conversion is in excellent agreement with the experimental value (see Tab. 1).

Table I. - Fitted data of $\left(\mathrm{Fe}(\mathrm{bt})(\mathrm{NCS})_{2}\right)_{2}$ bpym.

\begin{tabular}{|l|c|c|c|c|c|c|}
\hline & $\begin{array}{c}\Delta \\
(\mathrm{K})\end{array}$ & $\begin{array}{c}J_{\mathrm{av}} \\
(\mathrm{K})\end{array}$ & $\begin{array}{c}J \\
(\mathrm{~K})\end{array}$ & $\begin{array}{c}J_{\mathrm{AB}} \\
(\mathrm{K})\end{array}$ & $r_{\text {eff }}$ & $\begin{array}{c}\Delta S \\
\left(\mathrm{~J} . \mathrm{K}^{-1} \cdot \mathrm{mole}^{-1}\right)\end{array}$ \\
\hline Present model & 778 & -142 & - & 61 & 84 & 44 \\
Previous model [15] & 979 & - & -153 & 62 & 250 & 52 \\
\hline
\end{tabular}


The computed $f(T)$ curve leads to a threshold value $\Delta J_{\text {thres. }} \simeq 106 \mathrm{~K}$. On the other hand, all fits taking $\Delta J \leq 110 \mathrm{~K}$ were found to be equally good (Fig. 7a). For comparison, we also show in figure $7 \mathrm{~b}$ (dashed line) the conversion curve for a larger $\Delta J$-value. Since the larger acceptable value of $\Delta J$ is slightly larger than the threshold value, we cannot definitively rule out the occurrence of symmetry breakings. Besides, the measured variation of the specific heat [9] does not give evidence for any discontinuity, and its seems likely that there are no symmetry breakings in the studied compound.

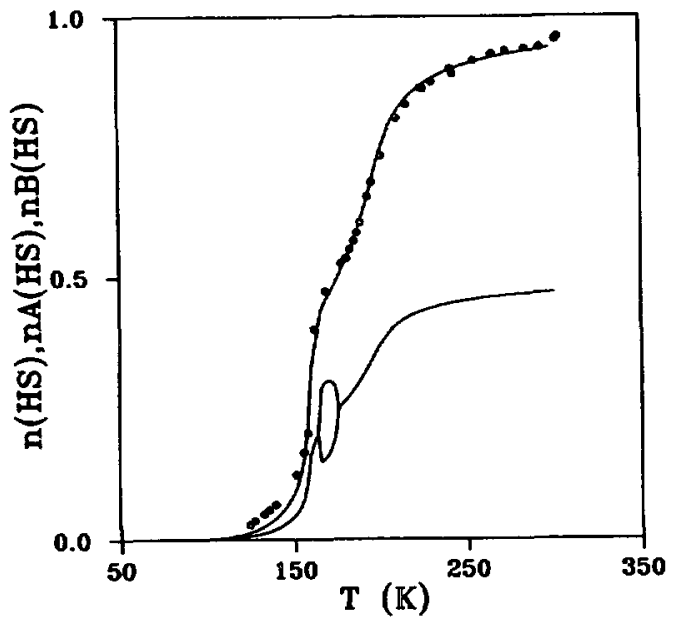

a)

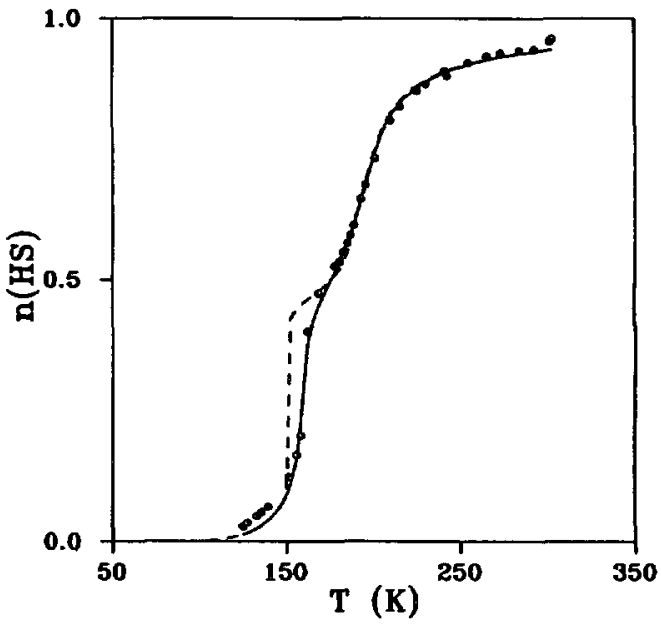

b)

Fig. 7. - Conversion curves computed with various $\Delta J$ values : $110 \mathrm{~K}(\mathrm{a}), 130 \mathrm{~K}$ (b, dashed line), to be compared to figure 6 .

\section{Conclusion.}

We have treated rigorously the intramolecular interaction of a bi-nuclear spin-crossover complex, while the intermolecular interactions were treated by the mean-field approach. The phase diagram of the model differs from that of the previous mean-field model in many respects ; the major feature concerns the occurrence of two-step conversions without symmetry breakings when the interaction difference $\Delta J$ is smaller than a threshold value depending on $J_{\mathrm{AB}}, J_{\mathrm{av}}$. The experimental two-step conversion of a binuclear complex has been adequately reproduced in close agreement with specific heat data with a difference $\Delta J$ presumably smaller than the threshold value.

\section{Acknowledgments.}

We are indebted to Phan Xuan Huy for technical assistance. 


\section{References}

[1] Koppen H., Muller E. W., Kohler C. P., Spiering H., Meissner E. and Gútlich P., Chem. Phys. Lett. 91 (1982) 348.

[2] Gutlich P. and Hauser A., Coord. Chem. Rev. 97 (1990) 1.

[3] Petrouleas V., Tuchagues J. P., Chem. Phys. Lett. 137 (1987) 21.

[4] Rakotonandrasana A., Boinnard A., Savariaud J. M., Tuchagues J. P., Petrouleas V., CARTIER C., Verdaguer M., Inorg. Chim. Acta 180 (1991) 19.

[5] Wajnflasz J. and PICK R., J. Phys. Colloq. France 32 (1971) C1-91.

[6] Wajnflasz J., Phys. Stat. Sol. 40 (1970) 537.

[7] Bari R., Sivardière J., Phys. Rev. B 5 (1972) 4466.

[8] Bousseksou A., Nasser J., Linarès J., Boukheddaden K. and Varret F., J. Phys. I France 2 (1992) 1381.

[9] Real J. A., Bolvin H., Bousseksou A., Dworkin A., Kahn O., Varret F. and ZarembowiTCH J., J. Am. Chem. Soc. 114 (1992) 4650.

[10] Slichter C. P., Drickamer H. G., J. Chem. Phys. 56 (1972) 2142.

[11] Sasaki N., Kambara T., Phys. Rev. B 40 (1989) 2442.

[12] SMART J., Effective field theory of magnetism (New York, 1966) pp. 37-45.

[13] Bousseksou A., Nasser J., Linarès J., Boukheddaden K., Varket F., Europ. Conf. Molecular Electronics (ECME'92, Padova, Aug. 92), Proceed. to appear in Molecular Crystals and Liquid Crystals.

[14] Takemoto J. H., Hutchinson B., Inorg. Nucl. Chem. Lett. 8 (1972) 769 ;

Gútlich P., Koppen H., Steinhauser H. G., Chem. Phys. Lett. 74 (1980) 3.

[15] Bousseksou A., Thèse de Doctorat de l'Université Paris 6, April 1992. 